\title{
A System-Social Approach to the Modeling of Corruption.
}

\author{
Alexander P. Mikhailov \\ Keldysh Institute of Applied Mathematics Russian Academy of Science \\ apmikhailov@yandex.ru \\ Evgeny A. Gorbatikov \\ Keldysh Institute of Applied Mathematics Russian Academy of Science \\ gor-104@mail.ru

\section{Elena D. Kornilina*} \\ Keldysh Institute of Applied Mathematics Russian Academy of Science \\ ekornilina@gmail.com
}

\section{Doi:10.5901/mjss.2013.v4n9p332}

\begin{abstract}
The proposed approach allows comparing strategies of struggle against corruption in hierarchies of arbitrary topology. Mathematically the comparison problem adds up to a relevant system of a large number of ordinary differential equations, or to a partial differential equation, and to corresponding discrete models. The underlined "Power-Society" model allows computing the level of power of instances in the hierarchy, which determines potential opportunities for corruption along with the behavior model of bureaucrats. In our study we limit our consideration to comparing the effectiveness of corruption suppression in different levels of hierarchy (from the "costs-vs-gains" viewpoint). Generally we found that the most profitable strategy consists in suppressing the lowest bureaucrats. A possibility of integration of the proposed system-social and game theory approaches into the general model is demonstrated.
\end{abstract}

\section{Introduction}

The phenomenon of corruption has adverse impacts on the economy and on society. It has been actively studied by mathematicians for nearly forty years: the first mathematical model dedicated to corrupt structures appeared in 1975 (see Rose-Ackerman, 1975). The debate about its' possible positive aspects have gone into the background: many studies have shown that corruption impacts negatively on a variety of economic and social aspects of life. Sergei Guriev (2004) shows that the presence of corruption leads to a greater number of bureaucratic checks caused by the fact that the officer is waiting for a bribe for their reduction. Corruption, of course, makes the economy less clear, as a bribe, being illegal, should be kept secret (Shleifer and Vishny (1993) develop this argument in detail). Ultimately, corruption slows economic growth, as it becomes more difficult to attract investment. This is confirmed by many empirical studies, in particular, the well-known work of Paolo Maoro (1995). A good overview of the work on mathematical modeling of corruption was made by Levin and Cyrik (1998).

In the vast majority of theoretical studies corruption is considered as an interaction of three (maximum four) agents. The standard paradigm of agents' rationality is used: the problem of maximizing the expected utility, which depends on the possible benefits and agents' attitude to risk, is solved.

The idea of our approach is that in addition to purely rational behavior, social power relations between agents should be considered as the agents' behavior is strongly dependent on the exercised power. Agent's power depends not only on monetary resources available, but also on possibility of influence on events, on her authority and also on other factors. In the presence of corruption the level of exercised power changes, and it is one of the factors that changes the agent's behavior. In addition, the extent to which the agent "resists" the corruption suppression depends on the level of her power. All these factors should be taken into account when modeling strategies of corruption suppression and searching the best among them.

The concept of power is difficult to determine and thus difficult to measure. However, basing on relatively simple assumptions, it is possible to create qualitative mathematical models with the explicit value of power. Such models 
provide an opportunity to consider the power relations and their results in hierarchies of any topology.

By specifying different characteristics of agents' behavior, a variety of scenarios describing the evolutions in the "Power-Society" model can be attained, including catastrophic ones - anarchy and totalitarianism. The model of power distribution in hierarchical structures was proposed by A.P. Mikhailov (1994). Detailed description of the model can be found in A.A. Samarskii and A.P. Mikhailov (2002), and also in Mikhailov (2006). The study of anti-corruption strategies based on this model was first made by Mikhailov (1997).

The described approach does not deny the rationalist tradition of game-theoretic study of corruption, but rather complements it. At the micro level, agents can follow the same principles of utility maximization when deciding on possible corruption level. However, at the macro level, their decision will influence the hierarchy as a whole. In our opinion, the synthesis of the two approaches will help solve problems of finding the optimal strategy of corruption suppression for each specific hierarchy topology, no matter how sophisticated it might be.

In the next section we formulate the basic assumptions of the general model of power-society interaction, and also we write equations, the model leads to. The third section is devoted to numerical experiments and comparison of corruption suppression strategies with different models of agents' behavior. The fourth section provides a method which allows to take the rational motives of agents into account at the micro level and thereby to introduce corruption endogenously. The findings are summarized in the conclusion.

\section{The model}

\subsection{The general "Power-Society" model}

The "Power-Society" model pays main attention to hierarchies - ordered sets of empowered institutions. The powers of the institutions in the hierarchy are restricted either by law or by statutes of the respective organizations.

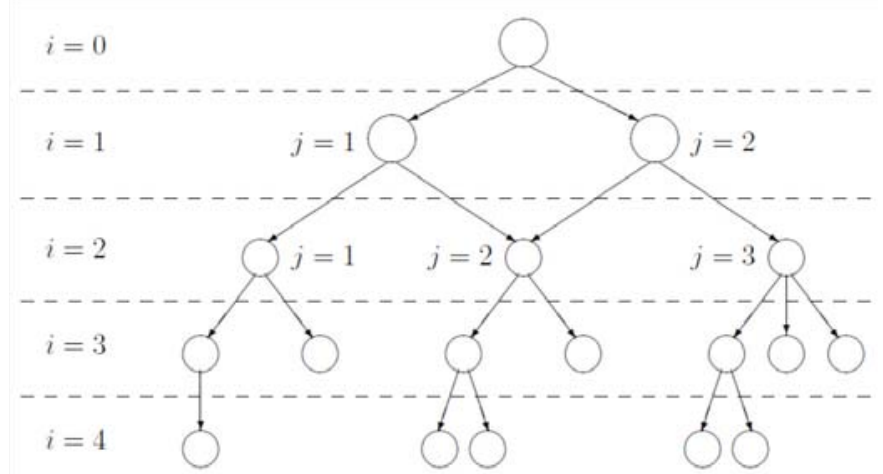

Figure 1. An example of the hierarchy considered. Each vertex of the graph corresponds to the number of the hierarchical level - $i$ and the vertex number in this level $-j$. The instance position in the hierarchy is thus characterized by a pair of indices $(i, j)$.

The interaction of instances in the hierarchy is performed by transmitting orders. Each instance receives orders from superiors and gives orders to its subordinates. An instance can in principle have several superiors, as well as several subordinates. The order delivery reduces the level of power of the instance which gave the order, and increases the level of power of the subordinate instance for the same amount. The level of power of instance $(i, j)$ is denoted as $p_{i j}(t)$. The number of orders per unit of time, i.e. a kind of "power flow", is supposed to be proportional to the difference in the levels of power of superior and subordinate. The proportionality coefficients for each pair of instances are denoted as $\kappa_{i j}(t)$. One possible interpretation of this assumption is the following: the less busy the subordinate instance is - the more orders per unit of time will come to it from its superiors. But subordinate instance can never give orders to his superior. This statement is called a hierarchical postulate.

The second agent in the model is a civil society. It is not involved in the transmission of orders, but may affect the 
level of power of any instance in the hierarchy. Being influenced by the civil society, the power level of each instance can be either expanded or narrowed. There are many ways of expressions society's response: from elections and referendums to rallies and strikes. The civil society's response is denoted as $F_{i j}(t)$. The interaction of the hierarchy and the civil society creates the final distribution of power, which is studied in the model, depending on various values of parameters.

We assume that there is some "perfect" level of power, in the eyes of the public authority, which should be exercised by the instance. The response brings the level of power to this "perfect" one, the stronger the farther is the real exercised level of power. Assuming the linear dependence, we write down the society response in the following way: $F=k_{i}\left(p_{i, j}^{0}-p_{i, j}\right)$. Here $p_{i, j}^{0}(t)$ is the "perfect" level of power, $k_{i}(t)$ is the proportionality coefficient. We call such type of society response legal, and this is the type of response we assume throughout this work.

The change of the level of power of instance $(i, j)$ during small period $\Delta t$ can be written as follows:

$$
\Delta p_{i, j}=\left(\sum_{k} \kappa_{i-1, k, j}\left(p_{i-1, k}-p_{i, j}\right)-\sum_{l} \kappa_{i, j, l}\left(p_{i, j}-p_{i+1, l}\right)+k_{i, j}\left(p_{i, j}^{0}-p_{i, j}\right)\right) \Delta t
$$

, where $\Delta p_{i, j}=p_{i, j}(t+\Delta t)-p_{i, j}(t)$, and the right hand side is taken at the moment $t+\xi \Delta t, \quad 0 \leq \xi \leq 1$. The first sum is the powers received from superiors, the second sum is orders transmitted to the subordinates, the last term is the society response.

If we write the balance (1) for each instance, we get the system of nonlinear algebraic equations that reflects the balance of power in the whole hierarchy. With $\Delta t$ approaching zero, we obtain a system of differential equations which describes the change of instances' power levels over time. It is this system of equations that lies in the base of the numerical investigation of the distribution of power for concrete hierarchies.

If, moreover, all the parameters of instances at the level $i$ depend only on the number of this level, the distribution of power in the hierarchy is described by the only equation:

$$
n(x) \frac{\partial p}{\partial t}=\frac{\partial}{\partial x}\left(\kappa(x) n(x) \frac{\partial p}{\partial x}\right)+n(x) k(x)\left(p^{0}(x)-p(x, t)\right), \quad 0<\mathrm{x}<1, \quad \mathrm{t} \geq \mathrm{t}_{0}
$$

(here a discrete coordinate of levels $i$ is replaced by of continuous coordinate $x, n(x)$ is the parameter which characterizes the degree of branching of the hierarchy. For integer values of $x$ it is equal to the number of instances at the corresponding level).

The solution of equation (2), or a system of equations of type (1) for each instance, with the relevant boundary conditions, gives power dynamics in the hierarchy.

In the general case the model results to a nonlinear integral-differential equation (see details in Mikhailov (2006)). In this paper we use a simplified model, which, however, retains all the main qualitative properties of the general one.

\subsection{Corrupted power structures.}

We understand the concept of corruption as a secret mercenary use of power on behalf of private groups. We divide corrupt activities into three types. The first type is the actions that, by law, have to be done, but are done for a bribe - are called usual corruption and are denoted by the index 0 . The example is corruption while getting driver's license or other documents. The second type is the actions that, by law, should not have been executed. Such corruption is called corruption of overactivity and is denoted by the index + . This type of corruption occurs, for example, when giving of licenses for enterprises which have not met some technological requirements. The third type is connected with failure to comply required actions. Such corruption is said to be a corruption of inactivity, and is denoted by the index -. An example would be a lack of fire inspections.

Corrupted officials can react differently to anti-corruption measures. We consider two types of their behavior:

1) The officials do not try to compensate their losses

2) The officials try to maintain the proportion of corrupt actions.

If only a certain type of corruption is suppressed, the official may retain the proportion of corrupted actions 
increasing his involvement in the other types of corruption. If there is no possibility to "switch" to the other type of corruption, the first and second types of behavior do not differ from each other.

For each of the two types of behavior, we introduce the concept of degrees of corruption $-c^{I}$ and $c^{I I}$, respectively. They are equal to the ratio of the number of actions performed for a bribe to the total number of actions in the law-abiding case - for the first type, and to the to the total number of actions performed without a bribe - for the second type. On the detailed motivation of such definition see Gorbatikov (2013).

It is clear that in the presence of corruption of overactivity or inactivity the power flow in the hierarchy is different from the no-corruption case. Equation (1) then becomes

$$
\Delta p_{i, j}=\left(\sum_{k} \kappa_{i-1, k, j}^{f}\left(p_{i-1, k}-p_{i, j}\right)-\sum_{l} \kappa_{i, j, l}^{f}\left(p_{i, j}-p_{i+1, l}\right)+k_{i, j}\left(p_{i, j}^{0}-p_{i, j}\right)\right) \Delta t
$$

where $\kappa_{i, j, k}^{f}=\left(1+c^{I}{ }_{+i, j}-c_{-i, j}^{I}\right) \kappa_{i, j, k}$ for the first type of officials' behavior and

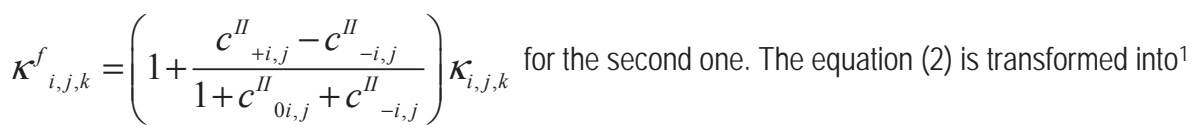

$$
n(x) \frac{\partial p}{\partial t}=\frac{\partial}{\partial x}\left(n(x) \kappa^{f}(x) \frac{\partial p}{\partial x}\right)+n(x) k\left(p^{0}(x)-p(x, t)\right), \quad 0<\mathrm{x}<1, \quad \mathrm{t} \geq \mathrm{t}_{0}
$$

It is evident, therefore, that the corruption does not affect the type of the equation and just changes the effective values of parameters $\kappa_{i, j}$.

It is assumed that the damage to society from the usual corruption is simply the amount of bribery (denoted $b$ with the corresponding index). Indeed, all orders must be given in the same manner as in the case of law-abiding, and the only difference is that the instance "picks up" a bribe from society for its actions. In the case of other types of corruption, in addition to the damage of a bribe, there is the damage from the very fact of performing illegal or not performing obligatory orders, and this damage (in monetary terms) can be much greater than the size of bribe. It is assumed that for these types of corruption damage equals to the entire spent / not spent resources of power, plus the actual size of the bribe. Full damage from corruption is equal to the sum of damages caused by all three forms of corruption.

A damage caused by the hierarchy as a whole is understood here as the damage divided by the total number of useful actions. The use is summed up from the actions performed without a bribe, and the actions carried out within the framework of the usual corruption. Then the relative harm from the actions of the hierarchy at a particular moment for the first type of behavior ${ }^{2}$ is as follows:

$$
D^{I}(t)=\left(\sum_{i, j}\left(c_{0}^{I} b_{0}+c_{-}^{I}\left(1+b_{-}\right)+c_{+}^{I}\left(1+b_{+}\right)\right) \sum_{l} \kappa_{i, j, l}\left(p_{i, j}-p_{i+1, l}\right)\right) \cdot\left(\left(1-c_{-}^{I}\right) \sum_{l} \kappa_{i, j, l}\left(p_{i, j}-p_{i+1, l}\right)\right)^{-1}
$$

In order to define the value of suppressing corruption in the instance we assume that it is

1. the higher, the higher the level of power of the particular instance is,

2. the higher, the higher the extent of corruption, that is, the value of bribes, is.

Then, the total relative cost of the complete suppression of corruption in the hierarchy is:

$$
V^{I}(t)=\left(\sum_{i, j}\left(c_{0}^{I} b_{0}+c_{-}^{I} b_{-}+c_{+}^{I} b_{+}\right) \varphi\left(p_{i, j}\right) \sum_{l} \kappa_{i, j, l}\left(p_{i, j}-p_{i+1, l}\right)\right) \cdot\left(\left(1-c_{-}^{I}\right) \sum_{l} \kappa_{i, j, l}\left(p_{i, j}-p_{i+1, l}\right)\right)^{-1}
$$

where $\varphi\left(p_{i, j}\right)$ is an increasing function ${ }^{3}$. The expressions for the relative damage and cost of corruption suppression in the continuous case are similar.

\footnotetext{
${ }^{1} \mathrm{~A}$ detailed derivation of the formulas is available in the appendix upon request.

${ }^{2}$ The expressions for the second behavior model are obtained in a similar manner

${ }^{3}$ In this work $\varphi\left(p_{i, j}\right)=\ln \left(1+p_{i, j}\right)$ is used. In Mikhailov (2006) this function was assumed linear.
} 
The parameter of strategies comparison - the effectiveness of corruption suppression - is defined as follows. Let $C$ be the degree of corruption in the hierarchy after the implementation of measures of the corruption suppression, $C+\Delta C$ be the degree of corruption before the measures. The efficiency of corruption suppression is defined as the ratio of the change of the relative damage to the change of the value of the complete corruption suppression in the hierarchy:

$$
E=\frac{D(C+\Delta C)-D(C)}{V(C+\Delta C)-V(C)}
$$

If the final situation is worse than the initial one, the effectiveness of anti-corruption measures is considered as non-defined.

\section{Numerical experiment.}

The model formulated in the previous section, allows answering a number of questions related to strategies of control and suppression of corruption. This section is dedicated to numerical studying of these questions for the concrete power structure.

As an example, we take a structure of Moscow police of 2012 year pattern. The main instance is the General Directorate of Internal Affairs. Departments of Internal Affairs are subordinate to it, one in each district. Subordinate to them, in turn, are the Department of the Interior, one in a few territories. District offices subject to them, and finally, each district office has several local policemen responsible for zones entrusted to them.

The corresponding system of equations obtained from (1) with the third type boundary condition is solved numerically. After this functionals of damages and costs are computed, and then according to (7) the efficiencies of different strategies of corruption suppression are compared ${ }^{4}$.

\subsection{Corruption on what hierarchical level should be suppressed first?}

Here we study the case of the presence of only one corruption type out of three. Are dealt with The cases of non-zero levels of three different corruption types: usual $\left(c_{0}\right)$, inactivity $\left(c_{-}\right)$and overactivity $\left(c_{+}\right)$- are considered in turn. In all cases, we assume that the initial hierarchy is corrupted uniformly.

Table 1 shows the results of the experiment for the model of the first behavior type. The results for the second type are qualitatively similar. Columns in the tables correspond to different strategies of corruption limitation: the first five columns - the complete liquidation of corruption at the level $i$, the last four - linear strategies with the indicated dependence of desired corruption level on the level of the hierarchy $i$.

\begin{tabular}{|c|c|c|c|c|c|c|c|c|c|}
\hline & Level 0 & Level 1 & Level 2 & Level 3 & Level 4 & $c=\frac{i}{8}$ & $c=\frac{4-i}{8}$ & $c=\frac{2-|2-i|}{4}$ & $c=\frac{|2-i|}{4}$ \\
\hline$c_{+}$ & 1,41 & 1,92 & 3,12 & 6,28 & 14,67 & 1,77 & 2,61 & 1,64 & 2,46 \\
\hline$c_{-}$ & 1,59 & 1,89 & 2,40 & 2,93 & 3,34 & 1,82 & 2,18 & 1,73 & 2,13 \\
\hline$c_{0}$ & 0,13 & 0,17 & 0,29 & 0,58 & 1,32 & 0,16 & 0,24 & 0,15 & 0,22 \\
\hline
\end{tabular}

Table 1: I type of behavior. In columns - different strategies of corruption limitation; in rows - the type of corruption considered. In cells - the effectiveness of appropriate strategies.

The main result is the following: struggle against corruption is mostly effective on the lower levels of hierarchy. Moreover, the combined strategies which include the highest level, are less effective than the ones which do not include it.

${ }^{4}$ The desired level of power for all instances of the last level is assumed to be 1 . For the results presented in the tables, $\kappa=0,01 ; \mathrm{k}=0,5$. Reasonableness of such values, as well as numerical experiments with other values of parameters, are discussed in Gorbatikov (2013). 


\subsection{What type of corruption should be suppressed first?}

Here we consider the existence of all types of corruption at once in the hierarchy ${ }^{5}$. Strategies of the complete liquidation of corruption of one certain type are compared, as well as strategies of liquidation each of them on the lower hierarchical level. The results are shown in Table 2.

\begin{tabular}{|l|l|}
\hline$c_{0}$ totally & 0,21 \\
\hline$c_{+}$totally & 2,55 \\
\hline$c_{-}$totally & 1,73 \\
\hline$c_{0}$ on the lowest level & 1,32 \\
\hline $\boldsymbol{C}_{+}$on the lowest level & 14,56 \\
\hline $\boldsymbol{C}_{-}$on the lowest level & 2,23 \\
\hline
\end{tabular}

\begin{tabular}{|l|l|}
\hline$c_{0}$ totally & --- \\
\hline$c_{+}$totally & 2,19 \\
\hline$c_{-}$totally & 2,08 \\
\hline$C_{0}$ on the lowest level & -- \\
\hline$C_{+}$on the lowest level & 15,54 \\
\hline$C_{-}$on the lowest level & 2,73 \\
\hline
\end{tabular}

Tables $2 \mathrm{a}, \mathrm{b}$ : I and II behavior models. In cells of the tables - efficiencies of corresponding strategies.

In the case of the first behavior model (officials do not respond to the policy by increasing corruption level of other corruption types), it is clear that the best solution is to suppress corruption of overactivity. Thereafter it is worthwhile to suppress corruption inactivity and finally the usual corruption. Furthermore, the table shows that even complete suppression of corruption of overactivity is better than suppression of corruption of inactivity on the lowest level. That is, in the case of limited funds for anti-corruption measures it is necessary to focus entirely on corruption of overactivity, and if it is for some reason impossible - on corruption of inactivity.

In the case of the second behavior model results are quite different. Such a situation becomes possible, when the suppression of some type of corruption not only fails to reduce the damage, but also worsen the state of affairs. This can be seen for the usual corruption suppression: the damage from actions of the whole hierarchy with usual corruption being suppressed (in the entire hierarchy or only on its lower level, lines 1 and 4 of Table $2 b$, respectively) exceeds the damage of its actions before suppression. Efficiency is not determined, and it is thus of no sense to implement suppression measures. This result has an intuitive interpretation: officials' benefit from various types of corruption is the same, but for society usual corruption is much less painful. Furthermore, it is clear that the suppression of corruption of overactivity is again more efficient than of inactivity corruption, but the difference is not so striking as in the case of the second behavior model.

\subsection{Other models of behavior}

Now we drop the assumption that the struggle against corruption in a particular instance does not affect corruption levels in other instances. Indeed, the strategy corruption suppression firstly at senior levels is usually argued by the fact that after this measure it is much easier to suppress corruption among subordinates. Public exposure of several high-level officials may be useful not only as a direct reduction of the amount of bribes, but also as an edification for lower-level officials, who recognize that they may suffer the same fate. Specifications considered further allow taking this argument into account.

\subsubsection{Imitating behavior model}

It is assumed that the corruption level of subordinates cannot exceed the one of superiors. In this case, the corruption suppression in the in the certain instance gives a greater effect: the level of corruption is "automatically" reduced among all subordinates, without increasing the cost of the measures.

\footnotetext{
${ }^{5}$ We assume initial corruption levels to be $c_{0}=c_{+}=c_{-}=0,5$
} 
Table 3 presents the results of numerical experiments similar to discussed above. Once again, we are interested in the following questions: what type of corruption should be fought primarily, and at on what levels of the hierarchy it should be done.

\begin{tabular}{|l|c|c|c|c|c|}
\hline & Level 0 & Level 1 & Level 2 & Level 3 & Level 4 \\
\hline$c_{+}$ & 5,41 & 3,74 & 5,11 & 7,74 & 14,79 \\
\hline$c_{-}$ & 4,22 & 3,18 & 3,95 & 4,18 & 3,67 \\
\hline$c_{0}$ & 0,50 & 0,34 & 0,48 & 0,67 & 1,29 \\
\hline
\end{tabular}

Table 3: imitative behavioral model

In this model, the suppression of corruption among senior officials is not always the least profitable strategy. On the contrary, for all three types of corruption, it is more effective than the suppression of the officials on the middle (second or third) level, and in the case of corruption of inactivity it is the most profitable among all strategies. In other words, corruption of inactivity should be liquidated from the top, while the rest types - from the bottom. In addition, it is clear for all three types of corruption that corruption liquidation on the second level is the least profitable strategy.

\subsubsection{Cautious behavior model}

More close to reality than the model from 3.3.1 is the following: the complete suppression of corruption at some level of the hierarchy leads to more careful behavior of adjacent levels and to reduction of their corruption levels by the relative value $r<1$. The next level reduces the level of corruption by $r^{2}$, the following - by $r^{3}$ and so forth. For example, when suppressing corruption of overactivity on the level $i=2$ from $c_{+}=0,5$ to $c_{+}=0$, then on levels $i=1$ and $i=3$ the level of corruption decreases to $c_{+}=0,5(1-r)$, and on levels $i=0$ and $i=4$ - to $c_{+}=0,5\left(1-r^{2}\right)$. As in the previous example, the funds are spent only for corruption suppression at a certain level, and changes in the rest levels are obtained for free. Qualitatively, the results for such a behavior are similar to the results obtained in the previous section. The most effective strategy is to suppress the junior officers, the least effective - to suppress the senior. Indeed, when $r=0$, this pattern turns to the discussed in the previous subsection. For values of $r$, tending to unity, it is also clear that the most effective strategy is to suppress corruption among junior officers.

\section{Endogenous corruption. Game theory approach}

In the model considered above the levels of corruption assumed to be exogenous, that is researcher should have stated them himself. In the previous section we stated certain levels of corruption and calculated the optimal (in the abovestated sense) strategy for the suppression of unlawful behavior. But in reality, the officials choose the type of behavior themselves, depending on how strong and effective the existing anti-corruption measures are. This section provides an example of introducing corruption into the "Power-Society" model endogenously, using game theory technique. In addition, the following model clarifies the role of the amount of funds allocated to anti-corruption measures. All other assumptions on the instances' behavior, amount of exercised power, etc. remain the same.

\subsection{The model}

For simplicity, we consider only the corrupted system of two instances: the superior and the subordinate. For this simplest case we write down and solve maximization problems of instances and supervising organization. The ideological generalization to hierarchies of arbitrary topology is quite easy.

Let the superior instance has the power $p_{1}$, the subordinate - $p_{2}$. Let $c_{1}$ and $c_{2}$ be the number of activities per unit of time corresponding to the usual corruption (other types of corruption are not considered here for simplicity). 
Corruption of instances is limited from above by the amount of bribes which it may require for the activities it produces. In the case of a complete absence of any checks was the level of corruption among the rational economic agents reaches maximum - they demand a bribe for any action. Then from the "Power-Society" model it follows that:

$$
c_{1} \leq \kappa\left(p_{1}-p_{2}\right) ; \quad \mathrm{c}_{2} \leq \kappa p_{2}
$$

In the right hand side of inequalities there are corresponding power flows which satisfy the third type boundary condition for the problem (1). Note that $p_{1}$ and $p_{2}$, being stationary solutions of the corresponding equations, are interconnected with each other through the number of parameters and cannot be arbitrary.

Suppose that for each unit of its activities the instance requires a bribe $b$. We do not propose a detailed interpretation of unit of activities, note only that it should be understood as a certain scope of authority. Thus, the instances' profits from their illegal activities equal to $b c_{1}$ and $b c_{2}$ correspondingly.

The second agent is the anti-corruption supervisor, whose purpose is to detect corruption in the instances. If the detected corruption level is $\tilde{c}$, then the fine $f(\tilde{c})$ is imposed to the instance. The final instances' problems look as follows:

$$
\begin{aligned}
& b c_{1}-f\left(\tilde{c}_{1}\right) \rightarrow \max _{c_{1}} \quad \text { s.t. } \mathrm{c}_{1} \leq \kappa\left(p_{1}-p_{2}\right) \\
& b c_{2}-f\left(\tilde{c}_{2}\right) \rightarrow \max _{c_{2}} \quad \text { s.t. } \mathrm{c}_{2} \leq \kappa p_{2}
\end{aligned}
$$

Now turn to the supervisor's problem. We assume that the supervisor is initially informed about true levels of corruption. Funds $W$ on corruption disclosure are provided, and they should be allocated among instances to suppress corruption properly. This allocation can be represented as the choice of measures' efficiency: let the supervisor choose probabilities of detecting unit of corruption in every instance. The higher the chosen probability - the more expensive the strategy is. Moreover, we assume, as before, that the detection of unit of corruption is the more expensive the more power the instance possess. Finally, the cost is proportional to $g(p) \cdot c \cdot p r o b$, where $g(p)$ characterizes how cost grows with power: $g^{\prime}(p)>0$.

The supervisor's aim is to find as many acts of corruption as possible, given $W$. Thus, the problem is as follows:

$$
\begin{aligned}
& \tilde{c}_{1}+\tilde{c}_{2}=c_{1} \text { prob }_{1}+c_{2} \text { prob }_{2} \rightarrow \max _{\text {probl, prob } 2 \in[0,1]} \\
& \text { s.t. } c_{1} \operatorname{prob}_{1} g\left(p_{1}\right)+\mathrm{c}_{2} \operatorname{prob}_{2} g\left(p_{2}\right)=W
\end{aligned}
$$

We consider the following sequential game. First, the budget $W$ is allocated for struggling against corruption. Then each instance in the hierarchy, knowing $W$, chooses its level of corruption. After that, based on available data, the supervisor chooses efficiencies of corruption suppression separately for each instance. Other information, as well as game structure, is common knowledge.

\subsection{Solution}

\subsubsection{Supervisor's problem}

It is easy to show that the supervisor states efficiencies of anti-corruption measures in the following way (assume for simplicity $g(p)=p)$ :

$$
\operatorname{prob}_{1}{ }^{*}=\max \left\{0, \frac{W-p_{2} c_{2}{ }^{*}}{p_{1} c_{1}{ }^{*}}\right\} ; \quad \operatorname{prob}_{2}{ }^{*}=\min \left\{1, \frac{W}{p_{2} c_{2}{ }^{*}}\right\}
$$

If the complete suppression of corruption at the subordinate instance is too expensive, then all the money is spent only on it. If there are a lot of funds, corruption at the lower level is suppressed completely, and the remaining money 
goes to struggling at the superior instance.

\subsubsection{The subordinate instance problem}

Assume a convex function of fine: $f(c)=A c^{2}$. Constant $A$ characterizes the scale of fines. The case of a concave function of fines can be considered similarly.

Consider possible cases, depending on the ratio of the parameters:

1) $\kappa p_{2} \leq \frac{W}{p_{2}}$. This inequality means that the funds provided are enough for complete corruption suppression in the lower instance, even if its level is as high as possible. Equilibrium levels of corruption are stated by the following relations:
a) if $\frac{b}{2 A}<\kappa p_{2}$ then $c_{2}^{*}=\frac{b}{2 A}$;
b) if $\frac{b}{2 A}>\kappa p_{2}$, then $c_{2}^{*}=\kappa p_{2}$

Thus, we see that even if the probability of detection is equal to 1 , there is a non-zero value of corruption. In addition, by increasing the budget corruption does not disappear gradually, but changes abruptly. However, if corruption is too profitable for the instance (high value of $\frac{b}{2 A}$ means greater bribes and lower fines), then even an effective measures do not lead to the desired result. The only solution in this case is the legislative change of fines, i.e. the value of $A$.

2) $\kappa p_{2}>\frac{W}{p_{2}}$. With such parameters relationship it cannot be said at once, if there are enough funds to complete corruption suppression at the lower level. The solution is the following:

a) if $\frac{b}{2 A}>\frac{W}{p_{2}}$, then $c_{2}^{*}=\kappa p_{2}$;

b) if $\frac{b}{2 A}<\frac{W}{p_{2}}$, then, depending on parameters values, both cases $c_{2}{ }^{*}=\kappa p_{2}$ and $c_{2}{ }^{*}=\frac{b}{2 A}$ are possible. The transition between the two occurs at the threshold value $W=W^{*}$, equal to $W^{*}=p_{2} \sqrt{\frac{\kappa p_{2} b}{A}-\left(\frac{b}{2 A}\right)^{2}}$. It is easy to check that $\frac{b p_{2}}{2 A}<W^{*}<\kappa p_{2}^{2}$, that is the threshold value lies within the specified limits.

Thus, even if the amount of allocated funds is not very large and is not able to cope with maximal possible level of corruption, increasing the budget can lead to much less equilibrium corruption level of the instance.

\subsubsection{The superior instance problem}

All funds that remain from the suppression of the lower instance corruption, the supervisor spends on the upper. The amount of these funds depends on the behavior of the lower instance and in the considered cases equals to, respectively:

In the case 1, a) $W_{1}=W-\frac{b p_{2}}{2 A}$; in the case $\left.1, \mathrm{~b}\right) W_{1}=W-\kappa p_{2}^{2}$; in the case $\left.2, \mathrm{a}\right) W_{1}=0$; in the case $2, \mathrm{~b}$ ) depending on parameters values, $W_{1}=W-\frac{b p_{2}}{2 A}$ or $W_{1}=0$. 
The problem now has the same form as that for the lower instance, we only should replace $W$ by $W_{1}$. Depending on the value of $p_{1}$ all variants of equilibrium are again possible for the upper instance. The exceptions are those cases when $W_{1}=0$. For these cases, it is obvious that $c_{1}=\kappa\left(p_{1}-p_{2}\right)$.

\subsubsection{Discussion}

From the solution we can see that if the budget for the corruption suppression is small, it does not motivate hierarchy to reduce bribery. On the contrary, if the officials know that large budget is provided for the fight against corruption, then they are interested in reducing the amount of bribes, and as a result it is possible that that the budget will not be fully spent. This means that it may make sense to announce a budget that excesses the real.

It should be kept in mind that the levels of power, which is directly influence on the equilibrium, are given by equations of the type (1) and depend on several parameters, in particular, from $k, p^{0}{ }_{1}, p^{0}{ }_{2}$, obviously not appearing in the solution of our model in this section. It would be interesting to consider how the instances' decisions about corruption level depend on these parameters.

The above example is just an illustration of the general mechanism, and in reality the problem should be solved for the two, but for a by far larger number of instances, for such hierarchies as considered in the previous section. In this case, it is not possible to solve the problem analytically, and the main research method is a computational experiment.

As in the original model, the corruption suppression in the lower level is more effective. In both models, it follows from the assumption that the cost of suppression is proportional to the level of the instance's power. Game-theoretical model, however, has several advantages over the original "Power-Society" model. First, it explicitly takes into account the budget provided to the struggle against corruption. Second, the level of corruption is not set exogenously, but is a solution of the corresponding maximization problem, reflecting the preferences of instances that form an hierarchy.

\section{Conclusion}

Using the "Power-Society" model qualitative answers to many questions regarding the suppression of corruption in the hierarchies of complicated structure have been received. For a particular hierarchy consisting of several thousand instances, the questions "on what hierarchical level should corruption be suppressed first" and "what type of corruption should be suppressed first" were answered, as well as how to act in case of different behavioral characteristics of instances. Summarizing shortly, the answers are: corruption of overactivity is most harmful, and, given restricted budget, should be suppressed first at the lowest levels of a hierarchy, as well as other types. The latter finding fails, though, if imitating behavior is given.

The integration of the micro-level game-theoretic maximization problem of instances and the supervisor seems promising. It is shown that in the simplest case, qualitative predictions of the modified and the original models are the same.

\section{References}

Gorbatikov, Evgeny. "Modeling of Corrupted Power Structures" Mathematical Modeling of Social Processes. Collected articles. Moscow, Maks-Press, 2013. Vol.15, p. 22-47 (in Russian)

Guriev, Sergei. "Red tape and corruption". Journal of Development Economics, 2004, vol. 73(2), p. 489-504.

Levin M.I., Cyrik M.L. "Corruption as an object of mathematical modeling", Economics and Mathematical Methods, 1998, vol.34(3) (in Russian)

Maoro, Paolo. "Corruption and Growth". Quarterly Journal of Economics, 1995, vol.110, issue 3, p. 681-712

Mikhailov A.P. "Mathematical Modeling of Power Distribution in State Hierarchical Structures Interacting with Civil Society", Proceedings of 14th IMACS World Congress, Atlanta, USA, 1994. Vol.II, p. 831-834

Mikhailov A.P. "Efficient Strategies of Corruption Suppression in State Power Hierarchies", Proceedings of 15th IMACS World Congress, Berlin, Germany, 1997. Vol.III, p.727-733

Mikhailov A.P. "Modeling of the "Power-Society" system", Moscow, Fizmatlit, 2006 - 144 pages (in Russian)

Rose-Ackerman, Susan. "The economics of corruption". Journal of Public Economics, 1975, vol. 4, issue 2, p. 187-203 
Samarskii A.A. , Mikhailov A.P. "Principles of Mathematical Modeling, Ideas, Methods, Examples.", Taylor and Francis, $2002-350$ pages.

Shleifer A., Vishny R.W. "Corruption”. Quarterly Journal of Economics, 1993, vol.108, issue 3, p. 599-617 